\title{
The impact of infectious diseases consultation on the management and outcomes of Pseudomonas aeruginosa bacteraemia in adults: a retrospective cohort study
}

Fabian Chiong ${ }^{1 *}$ (D) Mohammed S. Wasef ${ }^{2}$, Kwee Chin Liew ${ }^{3,4}$, Raquel Cowan ${ }^{3}$, Danny Tsai, ${ }^{5,6}$, Yin Peng Lee ${ }^{7,8}$, Larry Croft ${ }^{8,9}$, Owen Harris ${ }^{4}$, Stella May Gwini ${ }^{10}$ and Eugene Athan ${ }^{3,11}$

\begin{abstract}
Background: Pseudomonas aeruginosa bacteraemia (PAB) is associated with high mortality. The benefits of infectious diseases consultation (IDC) has been demonstrated in Staphylococcal aureus bacteraemia and other complex infections. Impact of IDC in PAB is unclear. This study aimed to evaluate the impact of IDC on the management and outcomes in patients with PAB.

Methods: This is a retrospective cohort single-centre study from 1 November 2006 to 29 May 2019, in all adult patients admitted with first episode of PAB. Data collected included demographics, clinical management and outcomes for PAB and whether IDC occurred. In addition, 29 Pseudomonas aeruginosa (PA) stored isolates were available for Illumina whole genome sequencing to investigate if pathogen factors contributed to the mortality.

Results: A total of 128 cases of PAB were identified, $71 \%$ received IDC. Patients who received IDC were less likely to receive inappropriate duration of antibiotic therapy (4.4\%; vs 67.6\%; $p<0.01)$, more likely to be de-escalated to oral antibiotic in a timely manner (87.9\% vs $40.5 \% ; p<0.01)$, undergo removal of infected catheter $(27.5 \%$ vs $13.5 \% ; p=$ $0.049)$ and undergo surgical intervention $(20.9 \%$ vs $5.4 \%, p=0.023)$ for source control. The overall 30 -day all-cause mortality rate was $24.2 \%$ and was significantly higher in the no IDC group in both unadjusted (56.8\% vs $11.0 \%$, odds ratio $[O R]=10.63, p<0.001$ ) and adjusted analysis (adjusted $O R=7.84 ; 95 \%$ confidence interval, 2.95-20.86). The genotypic analysis did not reveal any PA genetic features associated with increased mortality between IDC versus no IDC groups.
\end{abstract}

Conclusion: Patients who received IDC for PAB had lower 30-day mortality, better source control and management was more compliant with guidelines. Further prospective studies are necessary to determine if these results can be validated in other settings.

Keywords: Pseudomonas aeruginosa, Bacteraemia, Infectious diseases consultation, Mortality

\footnotetext{
*Correspondence: Fabian.Chiong@nt.gov.au

'Department of Medicine, Alice Springs Hospital, PO Box 2234, Alice Springs, NT 0871, Australia

Full list of author information is available at the end of the article
}

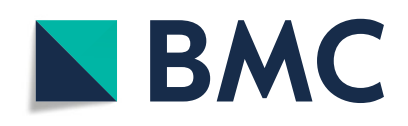

(- The Author(s). 2021 Open Access This article is licensed under a Creative Commons Attribution 4.0 International License, which permits use, sharing, adaptation, distribution and reproduction in any medium or format, as long as you give appropriate credit to the original author(s) and the source, provide a link to the Creative Commons licence, and indicate if changes were made. The images or other third party material in this article are included in the article's Creative Commons licence, unless indicated otherwise in a credit line to the material. If material is not included in the article's Creative Commons licence and your intended use is not permitted by statutory regulation or exceeds the permitted use, you will need to obtain permission directly from the copyright holder. To view a copy of this licence, visit http://creativecommons.org/licenses/by/4.0/. The Creative Commons Public Domain Dedication waiver (http://creativecommons.org/publicdomain/zero/1.0/) applies to the data made available in this article, unless otherwise stated in a credit line to the data. 


\section{Background}

Pseudomonas aeruginosa (PA) is a ubiquitous environmental Gram-negative pathogen that is responsible for many opportunistic and healthcare associated infections. Pseudomonas aeruginosa is the third most common Gram-negative pathogen causing bacteraemia in Australia [1]. It is also a common pathogen causing nosocomial infections such as pneumonia, postoperative wound infections and urinary tract infections (UTI) [2, 3]. Pseudomonas aeruginosa bacteraemia (PAB) is associated with prolonged hospital stays and has high mortality rates, particularly in neutropenic and immunocompromised hosts [1, 4]. It is clinically indistinguishable from other Gram-negative bacterial infections and given its intrinsic antimicrobial resistance, empiric therapy may be ineffective [5].

Data gathered across 36 institutions in Australia in the 2017 Australian Group on Antimicrobial Resistance (AGAR) study found that PA accounted for $8.8 \%$ of a total 7910 reported cases of Gram-negative bacteraemia [1]. Of the patients with PAB, 13.9\% had a length of stay of more than 30 days [1]. This is relative to $46.3 \%$ of patients with Enterobacterales bacteraemia who were discharged within 7 days [1]. The 30-day mortality for the cohort of patients with PAB was $20.7 \%$ [1]. Studies conducted prior to availability of effective therapies in the 1960s showed a mortality rate as high as 90\% [6, 7]. With the availability of more effective therapy, recent studies have shown mortality rates ranging between 18 and $61 \%[1,4,8-12]$.

Infectious diseases specialist consultation (IDC) is associated with improved clinical outcome in patients with Staphylococcal aureus bacteraemia, resulting in reduced mortality [13-17], lower treatment failure rates [18], better adherence to guidelines [13, 15], lower antimicrobial resistance development [15], higher probability of identifying a removable focus of bacteraemia $[14,16]$ and higher likelihood of patients receiving appropriate empiric and directed antimicrobial therapies for an appropriate duration $[14,15]$. Infectious diseases specialist consultation was also shown to reduce mortality in multidrug-resistant organism infection [19], candidemia $[20,21]$, cryptococcal infection [22] and enterococcal bacteraemia [23, 24].

In this retrospective study, we aimed to evaluate the impact of IDC on the clinical management and outcome of patients with $\mathrm{PAB}$, including the 30-day mortality. We also aimed to identify associations between 30-day mortality and patient factors, presence of IDC, source of $\mathrm{PAB}, \mathrm{PA}$ pathogen phenotypic and genotypic factors.

\section{Methods}

Ethics for this study was approved by the University Hospital Geelong Health Research and Ethics Committee (No.14/86).

\section{Study design}

We performed a retrospective study on consecutive adult patients admitted to the hospital with PAB from 1 November 2006 to 29 May 2019. In addition, 29 PA isolates from 29 patients which had been stored between 11 April 2017 and 29 May 2019 were examined to investigate whether pathogen factors contributed to the mortality, using Illumina Novaseq whole genome sequencing.

\section{Setting}

The University Hospital Geelong is the major regional tertiary-care centre for South Western Victoria, Australia, catering to a population of approximately 600, 000 people. It has 370 acute inpatient beds, an integrated Haematology, Oncology and Radiation Oncology service and a 24-bed, level 3, Intensive Care Unit (ICU).

\section{Patients}

All inpatients aged 18 years or over with at least one positive blood culture for PA during the study period were included. Detailed medical records for the corresponding admission were cross-referenced for data on age, gender, date of admission, date of diagnosis of PAB, date of discharge, date of death, underlying comorbidities, any invasive procedures (surgery, gastrointestinal and urological instrumentation) performed 30 days prior to positive blood culture for PA, source of their PAB (if known), appropriateness of their antimicrobial therapy, source control intervention, bedside consultation by infectious diseases (ID) specialist and 30-day mortality.

The pre-morbid and concurrent disease burden for each patient was assigned a value for severity using the Charlson comorbidity index [25]. This score was subsequently used to risk stratify the cohort and measure the impact of comorbid disease on 30-day mortality.

\section{Microbiological testing}

Blood cultures were incubated in BD BACTEC ${ }^{\mathrm{m}}$ Instrumented Blood Culture System and microorganisms from positive blood cultures were processed via standard method on horse blood, chocolate and MacConkey agar plates. Species identification was processed by Bruker MALDI-TOF or VITEK (bioMérieux) machine and antimicrobial susceptibility testing was processed by VITEK (bioMérieux) machine.

DNA extraction of this isolate was performed using the HiYieldTM Genomic DNA Mini Kit and Nextera library preps were made for each sample according to the standard protocol. The libraries were subjected to whole-genome sequencing using the NovaSeq 6000 Sequencer (Illumina) according to the manufacturer's instructions. Approximately 2Gb of 2x150nt paired-end reads were generated for each sample. Reads were cleaned 
and trimmed using fastp with default parameters. Snippy (https:/github.com/tseemann/snippy v4.6.0) was used to call SNPs based on the reference PA01 genome. SNP frequencies were extracted from the Snippy output with custom perl scripts and Fisher's exact test was run for each SNP contingency matrix using Matlab R2019b. Gene presence/absence across all samples was determined based on $95 \%$ identity over $90 \%$ of the length of the protein using Diamond blastp (v2.0.4). Presence/absence was counted per isolate and summed for the no IDC and IDC groups. A Fisher exact test was run in Matlab for each of the 177,085 proteins and variants to see if any were significantly present between the 2 groups.

\section{Clinical definitions}

- Pseudomonas aeruginosa bacteraemia episode was defined as a patient with consistent clinical illness having PA isolated from one or more of the aerobic or anaerobic bottles of their blood culture. The date of onset of PAB was defined as the date of the first positive blood culture for PA.

- Fever was defined as a single recorded body temperature $\geq 38^{\circ} \mathrm{C}$.

- A systolic blood pressure of less than $90 \mathrm{mmHg}$ was attributed to a presentation with shock.

- A patient was deemed to have febrile neutropenia if they had a temperature of $38^{\circ} \mathrm{C}$ or over and an absolute neutrophil count less than $0.5 \times 10^{9} / \mathrm{L}$.

- Health care associated infection was defined by a positive blood culture of PA more than $48 \mathrm{~h}$ after hospital admission, or 3 days after discharge, or less than 30 days after an invasive procedure.

- The criterion for being considered immunosuppressed was any form of chemotherapy or immunomodulatory medications (equivalent or more than $20 \mathrm{mg}$ of prednisolone a day) for longer than 4 weeks, patients with a clinical diagnosis of hematologic malignancy, or HIV infection, or has received solid organ or bone marrow transplantation.

- Duration of antibiotic treatment for PAB was dependent on the source of infection and appropriateness was assessed according to the Australian Therapeutic Guidelines: Antibiotic version 16, 2019 [26] and antimicrobial susceptibility testing. Compliance assessment was done by ID specialist, FC.

- De-escalation to oral antibiotic (Ciprofloxacin) in a timely manner was assessed according to the Australian Therapeutic Guidelines: Antibiotic version 16, 2019 [26]. Compliance assessment was done by ID specialist, FC.

- Heart failure was defined as left ventricular ejection fraction of $40 \%$ or less with at least class II New York Heart Association functional status.
- Liver cirrhosis was defined by the presence of radiological features of liver cirrhosis on abdominal ultrasound or documented diagnosis of liver cirrhosis in the patient's clinical notes.

- Renal insufficiency was defined as chronic kidney disease stage 3 or more using glomerular filtration rates and albumin creatinine ratio categories.

- Infectious diseases consultation included bedside consultation by a member of the ID service with entry of comments and recommendations for further clinical management were entered in the inpatient notes. Infectious diseases consultation was readily available and performed upon request from the primary service doctor with expectation for IDC to take place within $24 \mathrm{~h}$ of request.

\section{Outcomes}

Primary outcomes: Impact of IDC on all-cause 30-day mortality, clinical management and outcomes in patients with PAB.

Secondary outcome: Associations of 30-day all-cause mortality in patients with $\mathrm{PAB}$, including patient factors, presence of IDC, source of PAB, PA pathogen phenotypic and genotypic factors.

\section{Statistical analysis}

Categorical variables were reported as frequencies and percentages, continuous variables were summarised using median with interquartile range or mean with standard deviation. Patient characteristics considered were age (categorised using cut-offs of 65 and 75 years), Charlson comorbidity index (dichotomised using cutoff $=5$ ), febrile neutropenic status, immunosuppression status, haemodynamic status, presence of IDC, source of infection, appropriateness antibiotics therapy. Categorical variables were analysed with Fisher's exact test, and continuous variables were analysed with Wilcoxon ranksum test when appropriate. The relationship between patient characteristics and mortality were assessed using logistic regression and effects were reported as odds ratios (OR) with 95\% confidence interval. The adjusted logistic regression model accounted for variables that were of presumed clinically importance (age) and with $p<0.20$ in the unadjusted model. Survival rates in patients who received ID bedside consultation (+IDC) and those who did not (-IDC) were compared using the Log-rank test and cox proportional hazard model to account for confounding factors. Kaplan-Meier survival curves were constructed to illustrate the differences between the two groups. Logistic and cox regression adjusted for age, Charlson comorbidity index, IDC, surgical intervention and appropriateness of antimicrobial therapy. Fisher's exact test and correcting for multi-testing for single nucleotide polymorphisms analyses were 
undertaken using Stata Statistical Software version 15 (StataCorp, 2015. The College Press, Texas).

\section{Results}

\section{Demographics}

A total of 128 patients with $\mathrm{PAB}$ were identified, of whom 91 (71\%) received IDC and Table 1 summarises the demographic characteristics and present comorbidities. The median age of this cohort was 68 years old with almost an equal number of males and females. Baseline demographics of +IDC and -IDC were largely similar with exception for the proportion of patients with a Charlson comorbidity index of more than 5, which was larger in the -IDC group ( $54.9 \%$ vs $75.5 \%, p=0.048)$. The difference among individual comorbidities such as haematological malignancy, solid organ tumour, liver cirrhosis, renal insufficiency, heart failure, immunosuppression between the 2 groups were all less than $10 \%$ except for diabetes, which had $10 \%$ more in the - IDC group $(24.3 \%$ vs $14.3 \%$; $p=0.269)$. The admitting team distribution among the 2 groups is similar except for general medicine, where there was $11.5 \%$ more in the - IDC group $(32.4 \%$ vs $20.9 \%$; $p=0.248)$.

\section{Clinical management and outcomes}

Clinical management and outcomes observed for +IDC versus -IDC groups are presented in Table 2. Approximately half (49.2\%) of all the patients in this cohort required ICU admission, more in the -IDC group were admitted to ICU (62.2\% vs $44.0 \%, p=0.163)$. A total of 78 episodes $(60.9 \%)$ of PAB were hospital acquired and the +IDC group had 13.2\% more hospital-acquired PAB than the -IDC group (64.6\% vs $51.4 \%, p=0.248)$. The mean length of stay in hospital was longer in the +IDC group (16.7 vs 12.9 days, $p=0.248$ ) although the difference was not statistically significant. The + IDC group was less likely to receive inappropriate or no antibiotic $(26.4 \%$ vs $43.2 \%, p=0.06)$. The +IDC group was also less likely to have inappropriate duration of antibiotics treatment based on the source of infection and treatment of PAB was more frequently in line with the Australian Therapeutic Guidelines: Antibiotic [26] (4.4\% vs $67.6 \%, p<0.001)$. The +IDC group also had more than twice the proportion of the patients being de-escalated to oral antibiotic in a timely manner compared to the -IDC group $(87.9 \%$ vs $40.5 \% ; p<0.001)$.

Source of infection was identified in about two-thirds of patients with a slightly higher rate in the +IDC group $(69.2 \%$ vs $64.9 \%, p=0.630)$. Intravascular catheter was the most frequently identified source of $\mathrm{PAB}$ in the +IDC group, $12.4 \%$ more than the -IDC group (28.6\% vs $16.2 \%, p=0.216)$. The most commonly identified source in the -IDC group was pulmonary $(24.3 \%)$. Infected intravascular catheter was five times more likely

Table 1 Demographics of patients with Pseudomonas aeruginosa bacteraemia

\begin{tabular}{|c|c|c|c|c|}
\hline & $\begin{array}{l}\text { All patients } \\
(n=128)\end{array}$ & $\begin{array}{l}+ \text { IDC } \\
(n=91)\end{array}$ & $\begin{array}{l}\text {-IDC } \\
(n=37)\end{array}$ & $P$ Value \\
\hline Age (years): Median & 68 & 62 & 66 & 0.203 \\
\hline IQR & $59-78$ & $43-75$ & $47-77$ & \\
\hline Male: $n(\%)$ & $69(53.9)$ & $51(56.0)$ & $18(48.6)$ & 0.572 \\
\hline \multicolumn{5}{|l|}{ Comorbidities and medical history: $\mathrm{n}(\%)$} \\
\hline Charlson comorbidity index (severe, score $\geq 5$ ) & $78(60.9)$ & $50(54.9)$ & $28(75.7)$ & 0.048 \\
\hline Haematological malignancy & $46(35.9)$ & $35(38.5)$ & $11(29.7)$ & 0.465 \\
\hline Solid organ tumour & $28(21.9)$ & $18(19.8)$ & $10(27.0)$ & 0.507 \\
\hline Diabetes & $22(17.2)$ & $13(14.3)$ & $9(24.3)$ & 0.269 \\
\hline Liver cirrhosis & $15(11.7)$ & $12(13.2)$ & $3(8.1)$ & 0.612 \\
\hline Renal insufficiency & $25(19.5)$ & $20(22.0)$ & $5(13.5)$ & 0.396 \\
\hline Heart failure & $9(7.0)$ & $4(4.4)$ & $5(13.5)$ & 0.148 \\
\hline Immunosuppressed & $86(67.2)$ & $59(64.8)$ & $27(73.0)$ & 0.496 \\
\hline Admitting Teams: $\mathrm{n}(\%)$ & & & & 0.455 \\
\hline Hematology & $48(37.5)$ & $36(39.6)$ & $12(32.4)$ & \\
\hline General medicine & $31(24.2)$ & 19 (20.9) & $12(32.4)$ & \\
\hline Medical oncology & $26(20.3)$ & $18(19.8)$ & $8(21.6)$ & \\
\hline Renal medicine & $5(3.9)$ & $3(3.3)$ & $2(5.4)$ & \\
\hline Urology & $5(3.9)$ & $5(5.5)$ & $0(0)$ & \\
\hline Other $^{a}$ & $13(10.2)$ & $9(9.9)$ & $4(10.8)$ & \\
\hline
\end{tabular}

Abbreviations: $I Q R$ interquartile range, $+I D C$ received infectious diseases specialist consultation, $-I D C$ did not receive infectious diseases specialist consultation

a Other group includes General surgery, Gastroenterology, Cardiology, Cardiothoracic, Neurology, Orthopaedics, Radiation Oncology and Vascular teams 
Table 2 Clinical Management and Outcomes in Pseudomonas aeruginosa bacteraemia

\begin{tabular}{|c|c|c|c|c|}
\hline & $\begin{array}{l}\text { All patients } \\
(n=128)\end{array}$ & $\begin{array}{l}+ \text { IDC } \\
(n=91)\end{array}$ & $\begin{array}{l}- \text { IDC } \\
(n=37)\end{array}$ & $P$ Value \\
\hline 30-day mortality: n (\%) & $31(24.2)$ & $10(11.0)$ & $21(56.8)$ & $<0.001$ \\
\hline Intensive care unit admission: $\mathrm{n}(\%)$ & $63(49.2)$ & $40(44.0)$ & $23(62.2)$ & 0.163 \\
\hline Acquisition of PAB: $n$ (\%) & & & & 0.223 \\
\hline Community acquired & $50(39.1)$ & $32(35.2)$ & $18(48.6)$ & \\
\hline Hospital acquired & $78(60.9)$ & $59(64.8)$ & $19(51.4)$ & \\
\hline Length of stay in hospital (days; mean, standard deviation) & $15.5,7.8$ & $16.7,8.5$ & $12.9,5.7$ & 0.248 \\
\hline Inappropriate or no antibiotic $:$ n (\%) & $40(31.3)$ & $24(26.4)$ & $16(43.2)$ & 0.060 \\
\hline Inappropriate duration of antibiotic: $\mathrm{n}(\%)$ & $29(22.7)$ & $4(4.4)$ & $25(67.6)$ & $<0.001$ \\
\hline De-escalation to oral antibiotic in a timely manner: $\mathrm{n}(\%)$ & $95(74.2)$ & $80(87.9 \%)$ & $15(40.5 \%)$ & $<0.001$ \\
\hline Source of PAB: $n(\%)$ & & & & 0.786 \\
\hline Unidentified & $41(32.0)$ & $28(30.8)$ & $13(35.1)$ & \\
\hline Identified & $87(68.0)$ & $63(69.2)$ & $24(64.9)$ & \\
\hline Intravascular catheter & $32(25.0)$ & $26(28.6)$ & $6(16.2)$ & \\
\hline Pulmonary infection & $20(15.6)$ & $11(12.1)$ & $9(24.3)$ & \\
\hline Urinary tract infection & $18(14.1)$ & $14(15.4)$ & $4(10.8)$ & \\
\hline Skin and soft tissue infection & $13(10.2)$ & $10(11.0)$ & $3(8.1)$ & \\
\hline Other & $4(3.1)$ & $2(2.2)$ & $2(5.4)$ & \\
\hline Removal of infected catheter: $\mathrm{n}(\%)$ & $30(23.4)$ & $25(27.5)$ & $5(13.5)$ & 0.049 \\
\hline Surgical intervention for source control: $\mathrm{n}(\%)$ & $21(16.4)$ & $19(20.9)$ & $2(5.4)$ & 0.023 \\
\hline
\end{tabular}

Abbreviations: PA Pseudomonas aeruginosa, PAB Pseudomonas aeruginosa bacteraemia, $+I D C$ received infectious diseases specialist consultation, $-I D C$ did not

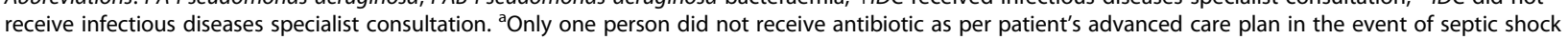

to be removed from the patient if the patient received IDC. Patients in +IDC group were also more likely to undergo surgical intervention to remove the source of infection or for source control for PAB (Table 2).

\section{Mortality}

A total of 31 of the 128 patients (24.2\%) died within 30days from the onset of $\mathrm{PAB}$, with a significantly higher mortality rate in the -IDC group (56.8\% vs $11.0 \%)$ (Table 2). After adjusting for other factors likely to influence 30-day all-cause mortality, the -IDC group was associated with 7-fold increased likelihood of death within 30 days from the time of PAB diagnosis. A pulmonary source was associated with higher rates of 30-day mortality (Table 3 ), even after controlling for other patient characteristics. P. aeruginosa bacteraemia resulting from an intravascular catheter, however, had an inverse association with 30-day mortality (OR $0.15, p=0.015$ ) (Table 3). Other associations with 30-day mortality (based on unadjusted analysis) are presented in Table 3. However, in the adjusted model, the effect of age and Charlson comorbidity index were attenuated (Table 3). Neither neutropenia nor immunosuppression were significantly associated with mortality. Kaplan-Meier survival curves (Fig. 1) clearly showed lower survival probability in patients with -IDC (unadjusted HR 7.45;
95\% CI 3.61-15.41; $p<0.001$ and adjusted $\mathrm{HR}=5.58$; 95\% CI 2.58-12.06; $p<0.001)$.

\section{Pathogen phenotypic and genotypic features}

All 128 PA isolates showed high proportion of susceptibility to anti-pseudomonal antibiotics. Antibiotic susceptibilities across this cohort of patients were: piperacillin/ tazobactam 89.9\%, ceftazidime 93.0\%, meropenem 96.9\%, gentamicin 95.3\%, and ciprofloxacin 96.1\% (Fig. 2). There were only 2 multidrug-resistant and one extensively drug-resistant PA isolates among all cases.

Phylogenomic analysis (Fig. 3) using all public PA genomes and the 29 sequenced isolates showed each PA isolate to be closest to a public genome geographically dispersed with worldwide distribution. Only one isolate was closest to a regionally derived public genome (Melbourne, Australia). This result suggested that there was no common source of patient infection and also the known diversity and lack of population structure found in small organisms, including eukaryotes. Each PA had up to 200 antibiotic resistance genes in its genome. Using a Fisher's exact test and correcting for multitesting for each of the 116,941 single nucleotide polymorphisms (SNPs), there was no SNPs with statistically significant allele frequency between the +IDC and -IDC groups or in terms of mortality. 
Table 3 Analysis of factors likely to influence 30-day all-cause mortality

\begin{tabular}{|c|c|c|c|c|c|}
\hline Variables & Mortality $\mathrm{n} /$ Total (\%) & Unadjusted OR (95\% Cl) & $P$ value & Adjusted $^{\mathrm{a}}$ OR $(95 \% \mathrm{Cl})$ & $P$ value \\
\hline \multicolumn{6}{|l|}{ IDC } \\
\hline Yes & 10/91 (11.0) & & & & \\
\hline No & $21 / 37(56.8)$ & 10.63(4.20-26.89) & $<0.001$ & $7.84(2.95-20.86)$ & $<0.001$ \\
\hline \multicolumn{6}{|l|}{ Age (years) } \\
\hline$<65$ & 10/53 (18.9) & & & & \\
\hline$\geq 65$ & $21 / 75(28.0)$ & $1.67(0.71-3.94)$ & 0.239 & $0.69(0.19-2.44)$ & 0.562 \\
\hline \multicolumn{6}{|l|}{ Age (years) } \\
\hline$<75$ & 15/86 (17.4) & & & & \\
\hline$\geq 75$ & $16 / 42(38.1)$ & $2.91(1.26-6.74)$ & 0.012 & $1.56(0.50-4.87)$ & 0.441 \\
\hline \multicolumn{6}{|l|}{ Gender } \\
\hline Female & $13 / 59(22.0)$ & & & & \\
\hline Male & $18 / 69(26.1)$ & $1.25(0.55-2.84)$ & 0.595 & $1.54(0.56-4.28)$ & 0.407 \\
\hline \multicolumn{6}{|l|}{ Charlson comorbidity index } \\
\hline Score $<5$ & $5 / 50(10.0)$ & & & & \\
\hline Score $\geq 5$ & 26/78 (33.3) & $4.50(1.59-12.75)$ & 0.005 & $5.14(1.22-21.58)$ & 0.025 \\
\hline \multicolumn{6}{|l|}{ Appropriate antibiotic therapy } \\
\hline Yes & $17 / 88(19.3)$ & & & & \\
\hline No & $14 / 40(35.0)$ & $0.44(0.19-1.03)$ & 0.059 & $0.38(0.06-2.26)$ & 0.289 \\
\hline \multirow[t]{2}{*}{ Surgical intervention for source control } & $2 / 21(9.5)$ & & & & \\
\hline & 29/107 (27.1) & $3.53(0.77-16.22)$ & 0.105 & $2.64(0.44-15.77)$ & 0.287 \\
\hline \multicolumn{6}{|l|}{ Febrile neutropenia } \\
\hline No & $22 / 83(26.5)$ & & & & \\
\hline Yes & $9 / 45(20.0)$ & $0.69(0.29-1.67)$ & 0.415 & $0.97(0.33-2.89)$ & 0.963 \\
\hline \multicolumn{6}{|l|}{ Immunocompromised } \\
\hline No & $11 / 45(24.4)$ & & & & \\
\hline Yes & 20/83 (24.1) & $0.98(0.42-2.29)$ & 0.965 & $0.64(0.19-2.14)$ & 0.474 \\
\hline \multicolumn{6}{|l|}{ Haemodynamic status at presentation } \\
\hline No shock & $2 / 63(3.2)$ & & & & \\
\hline In shock & 29/65 (44.6) & $24.57(5.50-109.76)$ & $<0.001$ & $45.77(10.30-203.41)$ & $<0.001$ \\
\hline \multicolumn{6}{|l|}{ Source of bacteraemia ${ }^{b}$} \\
\hline Pulmonary & $11 / 20(55.0)$ & $5.38(1.96-14.76)$ & 0.001 & $3.32(0.96-11.46)$ & 0.058 \\
\hline Invasive intravascular catheters & $2 / 32(6.3)$ & $0.15(0.03-0.69)$ & 0.015 & $0.18(0.04-0.90)$ & 0.036 \\
\hline Urinary tract & $1 / 18(5.6)$ & $0.16(0.02-1.24)$ & 0.079 & $0.17(0.02-1.32)$ & 0.091 \\
\hline Skin \& soft tissue & 2/13 (15.4) & $0.54(0.11-2.59)$ & 0.441 & $0.88(0.11-6.99)$ & 0.907 \\
\hline Unknown & $14 / 41(34.2)$ & $2.14(0.92-4.94)$ & 0.076 & $1.73(0.67-4.44)$ & 0.256 \\
\hline
\end{tabular}

Abbreviations: $O R$ odds ratio, $C l$ confidence interval, ID infectious diseases specialist

${ }^{\text {a }}$ Adjusted for age, Charlson comorbidity index, IDC, surgical intervention and appropriateness of antimicrobial therapy

$\mathrm{b}$ The odds ratio compares odds of death in current group with the odds of death in all other groups combined

\section{Discussion}

In our retrospective single-centre cohort study, not receiving an IDC was associated with a seven-fold increased risk of 30-day all-cause mortality. This finding is consistent with the results of another study on PAB which was conducted along with methicillin-resistant Staphylococcal aureus and candida blood stream infection, where -IDC was associated with 3.2 times increased hazard of death at 3 months and 5.3 times increased hazard of death in hospital compared with persons with IDC [27]. To the best of the authors' knowledge, the current study is the first that focuses specifically on the impact of IDC on the clinical management and outcomes for PAB.

In addition to the mortality benefit, this study also showed that IDC was associated with improved 


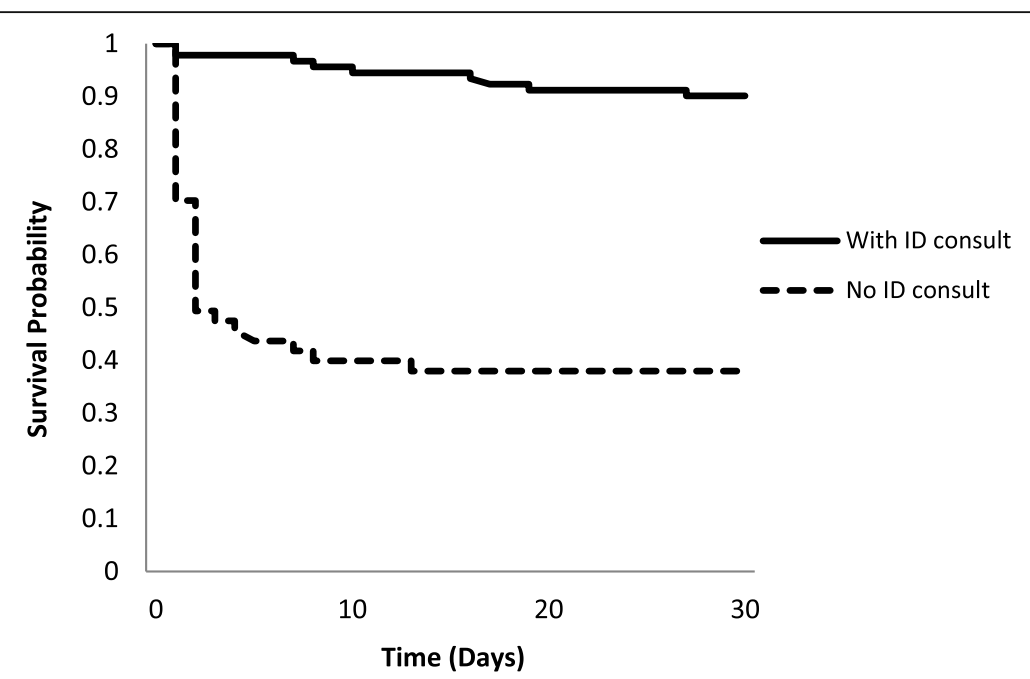

Fig. 1 Kaplan-Meier survival curve showing survival probabilities for patients with Pseudomonas aeruginosa bacteraemia with +IDC and -IDC

adherence to guidelines. Patients were less likely to receive inappropriate duration of antibiotic therapy according to the source of $\mathrm{PAB}$ and were more likely to be de-escalated to oral therapy in a timely manner. Even though IDC did not increase the likelihood of source identification of PAB, it was associated with better source control. Infected intravascular catheters were more likely to be removed and patients were more likely to have surgical intervention to address the source of $\mathrm{PAB}$ in the +IDC group. These factors could be the reasons for the positive impact of ID bedside consultation on the lower 30-day mortality rates in patients with PAB. These findings are also consistent with the benefits of IDC demonstrated in Staphylococcal aureus bacteraemia, where clinical management was more adherent to guidelines $[13,15]$ and higher probability of withdrawing a removable focus of bacteraemia $[14,16]$.

A previous study failed to demonstrate a mortality benefit of IDC in multidrug-resistant pseudomonal infection; however, patients with multidrug-resistant Pseudomonas were one of the smallest groups in the study and the study was underpowered to detect a possible mortality difference [19]. This study also did not specifically examine $\mathrm{PAB}$ and only multidrug-resistant pseudomonal infections [19]. Another study found that multiple IDC, not a single consultation, were associated

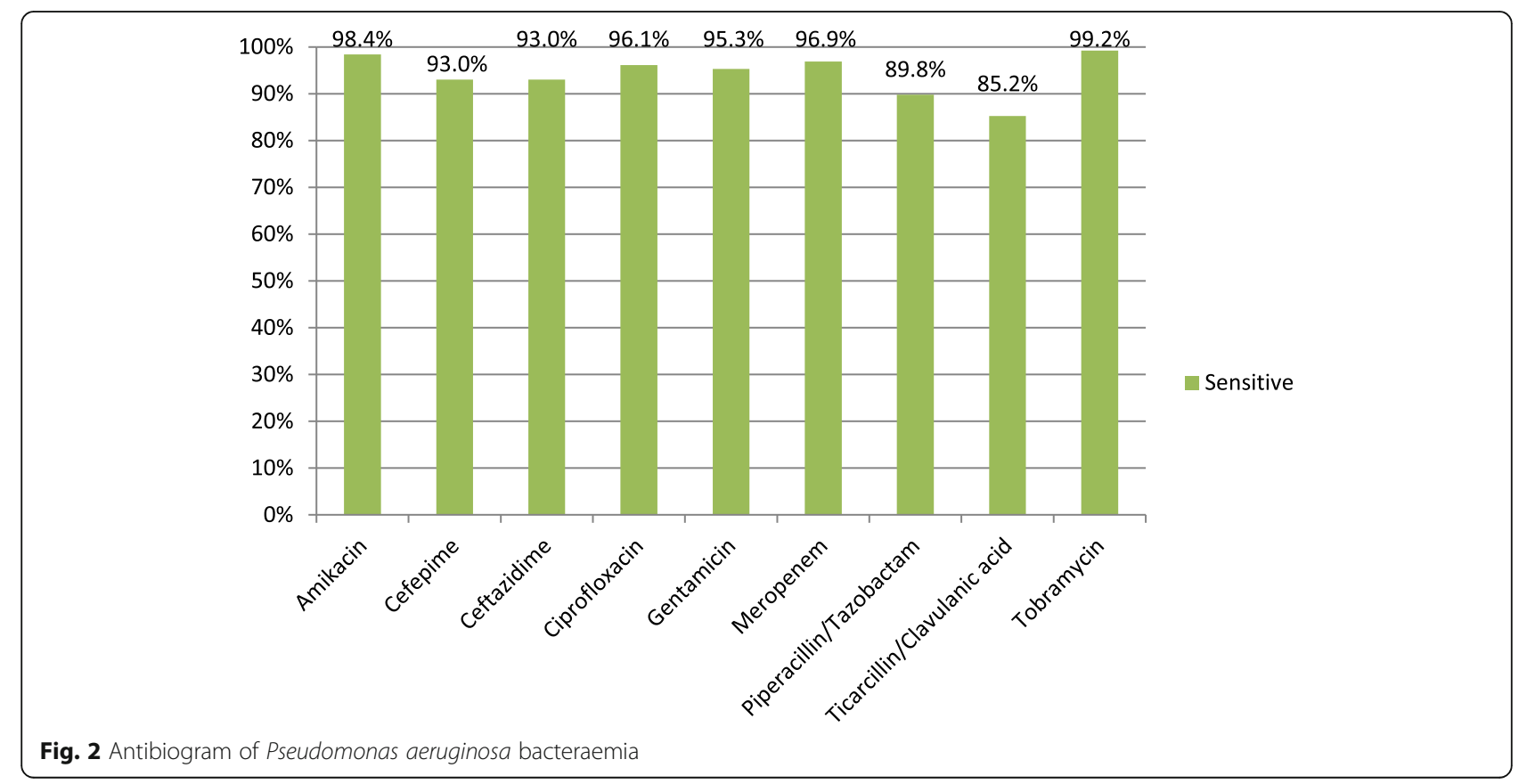




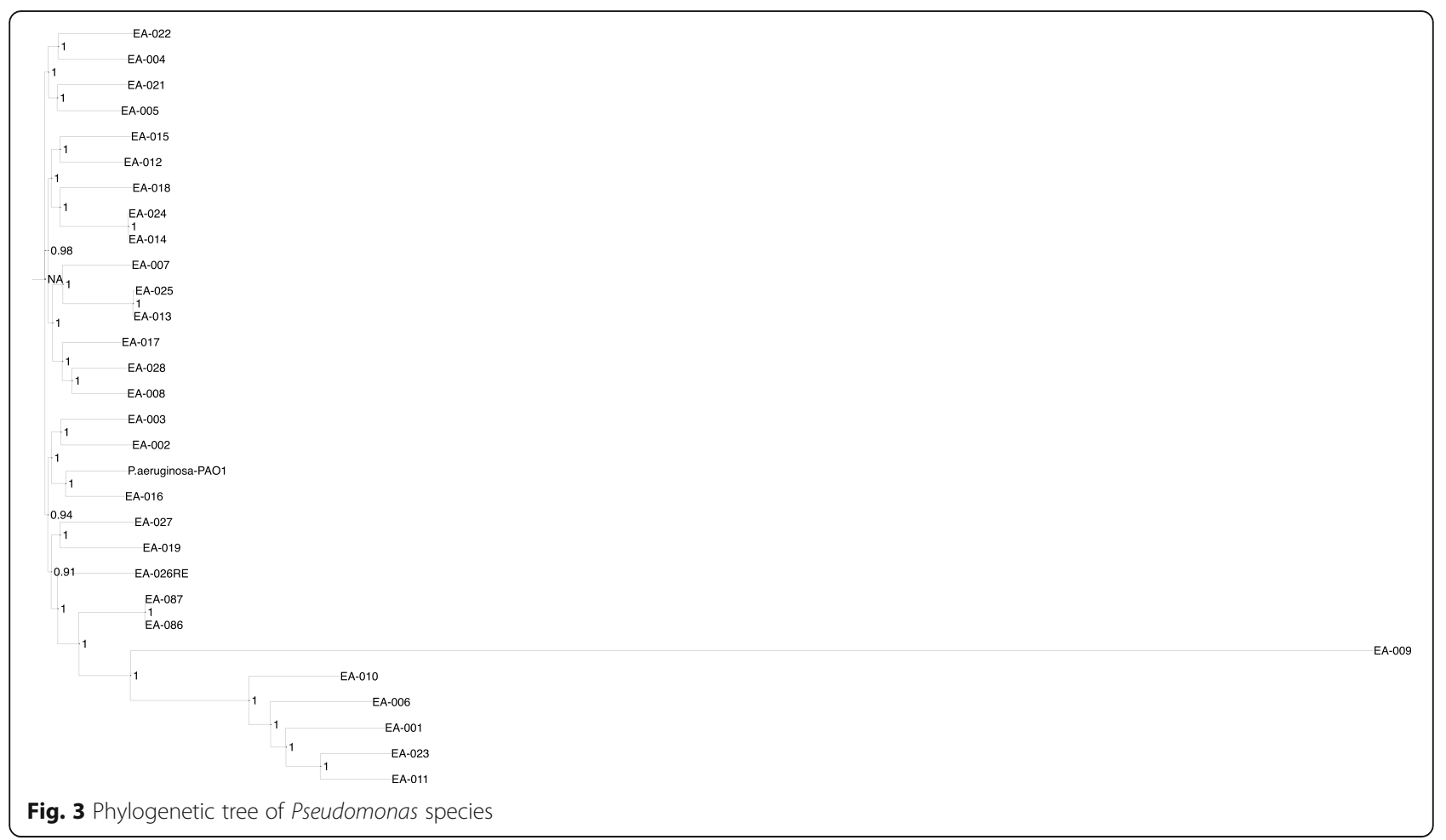

with lower mortality in bacteraemia secondary to both Gram-positive and Gram-negative pathogens [28]. Patients seen by ID specialists are usually more complex, one consultation may not be adequate to resolve the complex medical issues and thus it is crucial for ongoing follow-up with IDC to achieve better patient outcomes [29]. In addition to improved mortality, ID services in the healthcare system were also shown to improve transition of care from inpatient to outpatient based therapies, reduce hospital length of stay, reduce nosocomial infections and thereby lower health care expenditure [30].

We also tested for any pathogen genomic factors which could have affected the results. There were no statistically significant associations in the SNP allele frequencies for the two groups (+IDC versus -IDC and survived versus died), even though there was sufficient statistical power to find these if they were present. The lack of genomic markers showing differences between the groups strongly suggest that there is no genomic or strain which confounds the difference in mortality between the +IDC and -IDC groups.

We noted that the proportion of patients admitted to ICU and with Charleson comorbidity index score $\geq 5$ is greater in the -IDC group compared with the +IDC group. This could be explained by the regular multidisciplinary meetings between the ID and ICU teams, which occurred two to three times weekly during the study period. These meetings occurred as bedside discussion as part of the antimicrobial stewardship activities and not considered formal infectious diseases consultation. However, if the patient is deemed too complex for bedside advice, the ICU doctors could request a formal infectious diseases consultation. These multidisciplinary meetings may have reduced the frequency of formal IDC. Patients admitted under haematology, oncology and general medicine units comprise of the top three admitting teams for this cohort. Patients admitted under these teams usually have a higher Charlson's comorbidity score as most of the diagnoses in the Charlson comorbidity index are cared for under these three teams. There was also a once a week multidisciplinary meeting between the haematology, oncolology and ID teams. Many of the ID specialists also worked as general medicine specialists. Both of these factors could again reduce the frequency of IDC.

\section{Other associations of all-cause 30-day mortality}

Intravascular catheters were the primary source of infection for the highest identified proportion of the cases, at $25 \%$. The unadjusted results also showed a significantly lower mortality rate in this group. This low mortality was likely attributed to the fact that source control was easier to achieve with early removal of infected catheters. Pulmonary sources of $\mathrm{PAB}$ had the highest 30-day mortality risk. This is in line with the poor prognostic outcomes observed in patients with PA pneumonia in previous studies [31, 32] The two studies by Arancibia 
et al. and Charles et al. suggested that the high mortality rates observed in this group was likely due to the greater burden of disease, underlying severe obstructive airways disease and immunosuppression in individuals who were likely to contract opportunistic PA pneumonia.

Variables that we found with a significant association with all-cause 30-day mortality were similar to those previously reported. A 2003 study by Kang et al. identified severe sepsis, pneumonia, delay in starting effective antimicrobial therapy and high Acute Physiology And Chronic Health Evaluation II (APACHE II) score as significant risk factors contributing to increased mortality with PAB [33]. Another study identified ICU admission, coagulopathy, septic shock, and age greater than 65 years as key factors in increased mortality [34]. The presence of neutropenia was not an independent predictor of 30day mortality relative to other variables in this study. This is consistent with findings of 2 other PAB series in Korea and China [33, 35]. A possible explanation for this could be due to the rapidly evolving targeted immunotherapies for autoimmune conditions and cancers causing immune dysregulation that is not reflected by absolute neutrophil counts.

\section{Strength and limitations}

From our knowledge, this was the only study that has evaluated the PA pathogen genomic and phenotypic factors in assessing the clinical impact of IDC in PAB, acknowledging the lack of a priori hypothesis in terms of intrinsic pathogenicity factors in PA that hopefully could be addressed through future study. Genotypic analysis is costly and therefore we have only performed genomic studies on 29 frozen PAB isolates. Furthermore, frozen samples are only stored for 2 years in the hospital laboratory, hence isolates prior to April 2017 were not available for genotypic analysis.

This was a single-centre study in an Australian regional tertiary hospital with a limited sample size. The results may therefore not be generalised to other settings, particularly metropolitan tertiary centres with different patient case mix. Collaborating with other centres to achieve a larger cohort may strengthen our generalisability. However, the Barwon region's population is reasonably large and diverse to resemble the broader Caucasian Australian community [36] and University Hospital Geelong is the only tertiary centre in the region.

Patients with fulminant PA sepsis with high Sequential Organ Failure Assessment or APACHE II scores were at high risk mortality $[33,37,38]$, and they could have died prior to IDC. Due to the nature of a retrospective study, data on timing of IDC and early mortality were limited and they were not analysed. Madaline et al. described that early infectious disease consultation, within $12 \mathrm{~h}$ of ED triage, was associated with lower mortality in patients with severe sepsis or septic shock [39]. Given the clinical significance of this we suggest to further review the value of ID interventions on $\mathrm{PAB}$ in a prospective study.

It is possible that due to the retrospective study design, we were unable to account for unknown confounding variables. For example, the no IDC group in our cohort had higher proportion of patients with multiple comorbidities and this may have led to discrepancies in goals of care, which could possibly affect overall mortality irrespective of IDC. However, the adjusted models have included all possible confounders available in the retrospective dataset.

\section{Conclusion}

This study demonstrated the positive impact of IDC in the 30-day mortality, clinical management and outcomes of adult patients with PAB. Given the association between higher mortality rates and the absence of bedside IDC demonstrated in our study, we recommend that IDC be considered as standard of care for all adult PAB. Prospective studies on the impact of IDC in the management and outcomes $\mathrm{PAB}$, especially focusing on the timing and barriers to IDC, are warranted to determine if these results can be validated in larger cohort.

\section{Abbreviations \\ PA: Pseudomonas aeruginosa; PAB: Pseudomonas aeruginosa bacteraemia; ICU: Intensive Care Unit; ID: Infectious diseases; IDC: Infectious diseases consultation; +IDC: Received infectious diseases specialist consultation; -IDC: Did not receive infectious diseases specialist consultation}

\section{Acknowledgements}

Rodney Bird, Australian Clinical Laboratories, Geelong, Victoria, Australia data retrieval.

Radwanur Rahman Suman, Baker Institute of Research, Alice Springs, Northern Territory, Australia - initial statistical support.

Sanjeev Kumar Adhikari, Sivachandran Parimannan and Stella Loke, Deakin Genome Centre, School of Life and Environmental Sciences, University of Deakin, Victoria, Australia - genomic analysis.

\section{Authors' contributions}

FC: Principal investigator, conceptualization, data entry, data curation, data analysis, methodology, writing, editing, reviewing the manuscript. MSW: Data entry, writing original draft. KCL, YPL, LC: Library preparation and sequencing of 29 P. aeruginosa isolates, genotypic profile analysis, review and edit manuscript. $\mathrm{RQ}, \mathrm{EA}, \mathrm{OH}$ : Conceptualization, construction of database, provision of expert opinion, review and edit manuscript. DT, SG: Statistical analysis, review and edit manuscript. All authors have read and approved the final version of the manuscript.

\section{Funding}

The authors received no financial support for the research, authorship, and publication of this article.

Availability of data and materials

The datasets used and/or analysed in the study are available from the corresponding author on reasonable request. 


\section{Declarations}

\section{Ethics approval and consent to participate}

Ethics for this study was approved by the University Hospital Geelong Health Research and Ethics Committee (No.14/86). A waiver of patient consent was granted by the aforementioned Ethics Committee as this study is considered as a quality improvement project, retrospective in nature, and the manuscript does not include any identifiable data.

\section{Consent for publication}

Not applicable.

\section{Competing interests}

The authors have no competing/conflicting interests to declare in this article.

\section{Author details}

'Department of Medicine, Alice Springs Hospital, PO Box 2234, Alice Springs, NT 0871, Australia. ${ }^{2}$ Bankstown-Lidcombe Hospital, Sydney, Australia. ${ }^{3}$ Department of Infectious Diseases, University Hospital Geelong, Geelong, Australia. ${ }^{4}$ Australian Clinical Laboratories, Geelong, Australia. ${ }^{5}$ University of Queensland Centre for Clinical Research, University of Queensland, Brisbane, Australia. ${ }^{6}$ Rural and Remote Health NT, Flinders University, Alice Springs, NT, Australia. ${ }^{7}$ Deakin Genomic Centre, Deakin University, Geelong, Australia. ${ }^{8}$ School of Life and Environmental Sciences, Deakin University, Geelong, Australia. ${ }^{9}$ Asian Institute of Medicine, Science and Technology University, Bedong, Kedah, Malaysia. ${ }^{10}$ University Hospital Geelong, Geelong, Australia.

${ }^{11}$ School of Medicine Deakin University, Geelong, Australia.

\section{Received: 31 January 2021 Accepted: 28 June 2021}

Published online: 09 July 2021

\section{References}

1. Bell JM, Gottlieb T, Daley DA, Coombs GW. Australian group on antimicrobial resistance (AGAR) Australian gram-negative Sepsis outcome Programme (GNSOP) annual report 2017. Commun Dis Intell. 2018;2019:43.

2. Kim JM, Park ES, Jeong JS, Kim KM, Kim JM, Oh HS, et al. Multicenter surveillance study for nosocomial infections in major hospitals in Korea. Nosocomial infection surveillance Committee of the Korean Society for nosocomial infection control. Am J Infect Control. 2000;28(6):454-8. https:// doi.org/10.1067/mic.2000.107592.

3. Wisplinghoff $H$, Bischoff $T$, Tallent SM, Seifert $H$, Wenzel RP, Edmond MB. Nosocomial bloodstream infections in US hospitals: analysis of 24,179 cases from a prospective nationwide surveillance study. Clin Infect Dis. 2004;39(3): 309-17. https://doi.org/10.1086/421946.

4. Chatzinikolaou I, Abi-Said D, Bodey GP, Rolston KV, Tarrand JJ, Samonis G. Recent experience with Pseudomonas aeruginosa bacteremia in patients with cancer: retrospective analysis of 245 episodes. Arch Intern Med. 2000; 160(4):501-9. https://doi.org/10.1001/archinte.160.4.501.

5. Alikhani MY, Karimi Tabar Z, Mihani F, Kalantar E, Karami P, Sadeghi M, et al. Antimicrobial resistance patterns and prevalence of blaPER-1 and blaVEB-1 genes among ESBL-producing Pseudomonas aeruginosa isolates in west of Iran. Jundishapur J Microbiol. 2014;7(1):e8888. https://doi.org/10.5812/jjm.8888.

6. Schimpff SC, Greene WH, Young VM, Wiernik PH. Significance of Pseudomonas aeruginosa in the patient with leukemia or lymphoma. J Infect Dis. 1974;130 Suppl(0):S24-31.

7. Tapper ML, Armstrong D. Bacteremia due to Pseudomonas aeruginosa complicating neoplastic disease: a progress report. J Infect Dis. 1974;130 Suppl(0):S14-23.

8. Lodise TP Jr, Patel N, Kwa A, Graves J, Furuno JP, Graffunder E, et al. Predictors of 30-day mortality among patients with Pseudomonas aeruginosa bloodstream infections: impact of delayed appropriate antibiotic selection. Antimicrob Agents Chemother. 2007;51(10):3510-5. https://doi. org/10.1128/AAC.00338-07.

9. Maschmeyer G, Braveny I. Review of the incidence and prognosis of Pseudomonas aeruginosa infections in cancer patients in the 1990s. Eur J Clin Microbiol Infect Dis. 2000;19(12):915-25. https://doi.org/10.1007/s1 00960000410.

10. Siegman-lgra Y, Ravona R, Primerman H, Giladi M. Pseudomonas aeruginosa bacteremia: an analysis of 123 episodes, with particular emphasis on the effect of antibiotic therapy. Int J Infect Dis. 1998;2(4):211-5. https://doi.org/1 0.1016/S1201-9712(98)90055-8.
11. Todeschini G, Franchini M, Tecchio C, Meneghini V, Pizzolo G, Veneri D, et al. Improved prognosis of Pseudomonas aeruginosa bacteremia in 127 consecutive neutropenic patients with hematologic malignancies. Int J Infect Dis. 1998;3(2):99-104. https://doi.org/10.1016/s1201-9712(99)90017-6.

12. Vidal F, Mensa J, Almela M, Martinez JA, Marco F, Casals C, et al. Epidemiology and outcome of Pseudomonas aeruginosa bacteremia, with special emphasis on the influence of antibiotic treatment. Analysis of 189 episodes. Arch Intern Med. 1996;156(18):2121-6. https://doi.org/10.1001/a rchinte.1996.00440170139015.

13. Bai AD, Showler A, Burry L, Steinberg M, Ricciuto DR, Fernandes T, et al. Impact of infectious disease consultation on quality of care, mortality, and length of stay in Staphylococcus aureus bacteremia: results from a large multicenter cohort study. Clin Infect Dis. 2015;60(10):1451-61. https://doi. org/10.1093/cid/civ120.

14. Lahey T, Shah R, Gittzus J, Schwartzman J, Kirkland K. Infectious diseases consultation lowers mortality from Staphylococcus aureus bacteremia. Medicine (Baltimore). 2009;88(5):263-7. https://doi.org/10.1097/MD.0b013 e3181b8fccb.

15. Rieg S, Kupper MF. Impact of an infectious diseases consultation service on the quality of care and the survival of patients with infectious diseases. Z Evid Fortbild Qual Gesundhwes. 2015;109(7):500-10. https://doi.org/10.1016/ j.zefq.2015.09.008.

16. Saunderson RB, Gouliouris T, Nickerson EK, Cartwright E, Kidney A, Aliyu SH, et al. Impact of routine bedside infectious disease consultation on clinical management and outcome of Staphylococcus aureus bacteraemia in adults. Clin Microbiol Infect. 2015;21(8):779-85. https://doi.org/10.1016/j.cmi.2015.05.026.

17. Vogel M, Schmitz RP, Hagel S, Pletz MW, Gagelmann N, Scherag A, et al. Infectious disease consultation for Staphylococcus aureus bacteremia - a systematic review and meta-analysis. J Inf Secur. 2016;72(1):19-28.

18. Jenkins TC, Price CS, Sabel AL, Mehler PS, Burman WJ. Impact of routine infectious diseases service consultation on the evaluation, management, and outcomes of Staphylococcus aureus bacteremia. Clin Infect Dis. 2008; 46(7):1000-8. https://doi.org/10.1086/529190.

19. Burnham JP, Olsen MA, Stwalley D, Kwon JH, Babcock HM, Kollef MH. Infectious diseases consultation reduces 30-day and 1-year all-cause mortality for multidrug-resistant organism infections. Open Forum Infect Dis. 2018;5(3):ofy026

20. Kobayashi T, Marra AR, Schweizer ML, Ten Eyck P, Wu C, Alzunitan M, et al. Impact of infectious disease consultation in patients with candidemia: a retrospective study, systematic literature review, and meta-analysis. Open Forum Infect Dis. 2020;7(9):ofaa270.

21. Lagrou K, Van Wijngaerden E. Infectious disease consultation lowers candidaemia mortality. Lancet Infect Dis. 2019;19(12):1270-2. https://doi. org/10.1016/S1473-3099(19)30522-5.

22. Spec A, Olsen MA, Raval K, Powderly WG. Impact of infectious diseases consultation on mortality of Cryptococcal infection in patients without HIV. Clin Infect Dis. 2017;64(5):558-64. https://doi.org/10.1093/cid/ciw786.

23. Furuichi $M$, Furuichi $M$, Horikoshi $Y$, Miyairi I. Infectious diseases consultation improves treatment and decreases mortality by Enterococcal bacteremia in children. Pediatr Infect Dis J. 2018;37(9):856-60. https://doi.org/10.1097/INF. 0000000000001919

24. Lee RA, Vo DT, Zurko JC, Griffin RL, Rodriguez JM, Camins BC. Infectious diseases consultation is associated with decreased mortality in enterococcal bloodstream infections. Open Forum Infect Dis. 2020;7(3):ofaa064.

25. Charlson ME, Pompei P, Ales KL, MacKenzie CR. A new method of classifying prognostic comorbidity in longitudinal studies: development and validation. J Chronic Dis. 1987;40(5):373-83. https://doi.org/10.1016/0021-9681(87)90171-8.

26. Kelly H, editor. Therapeutic Guidelines - Antibiotic 16 ed. Melbourne, Australia 2019.

27. Chesdachai S, Kline S, Helmin D, Rajasingham R. The effect of infectious diseases consultation on mortality in hospitalized patients with methicillinresistant Staphylococcus aureus, candida, and pseudomonas bloodstream infections. Open Forum Infect Dis. 2020;7(1):ofaa010.

28. Tang G, Huang L, Zong Z. Impact of infectious disease consultation on clinical management and outcome of patients with bloodstream infection: a retrospective cohort study. Sci Rep. 2017;7(1):12898. https://doi.org/10.103 8/s41598-017-13055-2.

29. Tonelli M, Wiebe N, Manns BJ, Klarenbach SW, James MT, Ravani P, et al. Comparison of the complexity of patients seen by different medical subspecialists in a universal health care system. JAMA Netw Open. 2018;1(7): e184852. https://doi.org/10.1001/jamanetworkopen.2018.4852. 
30. McQuillen DP, MacIntyre AT. The value that infectious diseases physicians bring to the healthcare system. J Infect Dis. 2017;216(suppl_5):S588-S93.

31. Arancibia F, Bauer TT, Ewig S, Mensa J, Gonzalez J, Niederman MS, et al. Community-acquired pneumonia due to gram-negative bacteria and pseudomonas aeruginosa: incidence, risk, and prognosis. Arch Intern Med. 2002;162(16):1849-58. https://doi.org/10.1001/archinte.162.16.1849.

32. Charles PG, Whitby M, Fuller AJ, Stirling R, Wright AA, Korman TM, et al. The etiology of community-acquired pneumonia in Australia: why penicillin plus doxycycline or a macrolide is the most appropriate therapy. Clin Infect Dis. 2008;46(10):1513-21. https://doi.org/10.1086/586749.

33. Kang Cl, Kim SH, Kim HB, Park SW, Choe YJ, Oh MD, et al. Pseudomonas aeruginosa bacteremia: risk factors for mortality and influence of delayed receipt of effective antimicrobial therapy on clinical outcome. Clin Infect Dis. 2003;37(6):745-51. https://doi.org/10.1086/377200.

34. Aliaga L, Mediavilla JD, Cobo F. A clinical index predicting mortality with Pseudomonas aeruginosa bacteraemia. J Med Microbiol. 2002;51(7):615-9. https://doi.org/10.1099/0022-1317-51-7-615.

35. Zhang Q, Smith JC, Zhu Q, Guo Z, MacDonald NE. A five-year review of Pseudomonas aeruginosa bacteremia in children hospitalized at a single center in southern China. Int J Infect Dis. 2012;16(8):e628-32. https://doi. org/10.1016/j.jiji.2012.03.014.

36. Pasco JA, Nicholson GC, Kotowicz MA. Cohort profile: Geelong osteoporosis study. Int J Epidemiol. 2012;41(6):1565-75. https://doi.org/10.1093/ije/dyr148.

37. Singer M, Deutschman CS, Seymour CW, Shankar-Hari M, Annane D, Bauer M et al. The third international consensus definitions for Sepsis and septic shock (Sepsis-3). JAMA. 2016:315(8):801-10. https://doi.org/10.1001/jama.2016.0287.

38. Babich T, Naucler P, Valik JK, Giske CG, Benito N, Cardona R, et al. Risk factors for mortality among patients with Pseudomonas aeruginosa bacteraemia: a retrospective multicentre study. Int J Antimicrob Agents. 2020;55(2):105847. https://doi.org/10.1016/j.ijantimicag.2019.11.004.

39. Madaline T, Wadskier Montagne F, Eisenberg R, Mowrey W, Kaur J, Malik M, et al. Early infectious disease consultation is associated with lower mortality in patients with severe sepsis or septic shock who complete the 3-hour sepsis treatment bundle. Open Forum Infect Dis. 2019;6(10):ofz408.

\section{Publisher's Note}

Springer Nature remains neutral with regard to jurisdictional claims in published maps and institutional affiliations.

Ready to submit your research? Choose BMC and benefit from:

- fast, convenient online submission

- thorough peer review by experienced researchers in your field

- rapid publication on acceptance

- support for research data, including large and complex data types

- gold Open Access which fosters wider collaboration and increased citations

- maximum visibility for your research: over $100 \mathrm{M}$ website views per year

At $\mathrm{BMC}$, research is always in progress.

Learn more biomedcentral.com/submissions 